\title{
Grupo estratégico de promoção da saúde como dispositivo de cuidado a pessoas que vivem com diabetes mellitus
}

\author{
Group promotion of strategic health care as a device to persons living with diabetes mellitus \\ Grupo estratégico de promoción de la salud como dispositivo de atención a personas que viven con \\ diabetes mellitus
}

Recebido: 29/03/2021 | Revisado: 09/04/2021 | Aceito: 11/04/2021 | Publicado: 21/04/2021

Juliana Iscarlaty Freire de Araújo

ORCID: https://orcid.org/0000-0003-3379-7602

Universidade Federal do Rio Grande do Norte, Brasil

E-mail: ju.iscarlaty@ hotmail.com

Marília Rute de Souto Medeiros

ORCID: https://orcid.org/0000-0003-1817-6859

Universidade Federal do Rio Grande do Norte, Brasil

E-mail: mariliarute@hotmail.com

Talita Araújo de Souza

ORCID: https://orcid.org/0000-0002-3138-6626 Universidade Federal do Rio Grande do Norte, Brasil E-mail: talitaaraujo23@hotmail.com

Ilisdayne Thallita Soares da Silva

ORCID: https://orcid.org/0000-0003-2421-8090 Universidade Federal do Rio Grande do Norte, Brasil

E-mail: ilisdayne@yahoo.com.br

José Adailton da Silva

ORCID: https://orcid.org/0000-0002-6037-7649 Universidade Federal do Rio Grande do Norte, Brasil E-mail: Adailton.silva@ufrn.br

\begin{abstract}
Resumo
Este estudo tem por objetivo analisar as experiências dos sujeitos que convivem com diabetes e que participaram de um grupo com estratégias delimitadas, em relação ao controle da doença e exercício da autonomia pessoal. Trata-se de um estudo do tipo participativo, com abordagem qualitativa, apoiada na reflexibilidade, onde os encontros realizados deram origem a narrativas que foram organizadas em eixos temáticos e analisados. $\mathrm{O}$ trabalho mostrou que o grupo assume uma característica de rede de apoio, e agente facilitador para o convívio com a síndrome, onde a premissa da troca de vivências contribui significativamente no processo de transformação do outro, refletindo no processo de coconstrução da autonomia pessoal, e por consequência melhora da qualidade d vida dos sujeitos envolvidos. Pode-se concluir que as práticas grupais como dispositivo de promoção da saúde, possibilitam um impacto positivo na qualidade de vida das pessoas, desenvolvimento da autonomia e autocuidado.
\end{abstract}

Palavras-chave: Diabetes mellitus; Autonomia pessoal; Promoção da saúde.

\begin{abstract}
This study aims to analyze the experiences of subjects who live with diabetes and who participated in a group with defined strategies, in relation to disease control and exercise of personal autonomy. This is a participatory study, with a qualitative approach, supported by reflexivity, where the meetings held gave rise to narratives that were organized in thematic axes and analyzed. The work showed that the group assumes the characteristic of a support network, and a facilitating agent for living with the syndrome, where the premise of exchanging experiences contributes significantly to the process of transforming the other, reflecting on the process of co-construction of personal autonomy, and consequently improving the quality of life of the subjects involved. It can be concluded that group practices as a health promotion device, they enable a positive impact on people's quality of life, development of autonomy and selfcare.
\end{abstract}

Keywords: Diabetes mellitus; Personal autonomy; Health promotion.

\section{Resumen}

Este estudio tiene como objetivo analizar las experiencias de sujetos que viven con diabetes y que participaron en un grupo con estrategias definidas, en relación con el control de la enfermedade y el ejercicio de la autonomía personal. Se trata de un estudio participativo, con enfoque cualitativo, apoyado en la reflexividad, donde los encuentros realizados dieron lugar a narrativas que se organizaron en ejes temáticos y se analizaron. El trabajo mostró que el 
grupo asume la característica de una red de apoyo, y un agente facilitador para vivir con el síndrome, donde la premisa de intercambiar experiencias contribuye significativamente al proceso de transformación del otro, reflexionando sobre el proceso de co-construcción de la autonomía personal, y consecuentemente mejorando la calidad de vida de los sujetos involucrados. Se puede concluir que las prácticas grupales como dispositivo de promoción de la salud, posibilitan un impacto positivo en la calidad de vida de las personas, el desarrollo de la autonomía y el autocuidado.

Palabras clave: Diabetes mellitus; Autonomía personal; Promoción de la salud.

\section{Introdução}

A Diabetes Mellitus (diabetes) caracteriza-se como uma das Doença Crônica Não-Transmissível (DCNT), sendo um dos mais importantes problemas de saúde pública mundial (Sociedade Brasileira de Diabetes - SBD, 2020). Caracterizada como uma síndrome metabólica, a diabetes apresenta etiologia heterogênea representada pelo quadro de hiperglicemia persistente e distúrbios no metabolismo dos carboidratos, proteínas e gorduras, resultando na deficiência da produção ou da ação do hormônio insulina (SBD, 2020; Ministério da Saúde - MS, 2013).

Segundo a International Diabetes Federation (IDF), em 2019, cerca de 500 milhões de pessoas no mundo vivem com a diabetes, sendo em sua maioria adultos entre 20 e 79 anos (International Diabetes Federation-IDF, 2019).

Em 2019, o Brasil ocupava o $5^{\circ}$ lugar em relação aos países com maior número de pessoas que vivem com a diabetes, sendo 16,8 milhões de pessoas, estimando-se 26 milhões de pessoas até o ano de 2045 (IDF, 2019). No ano de 2015, cerca de $12 \%$ dos gastos públicos com saúde foram associados ao tratamento da diabetes ou de complicações relacionadas a ela (IDF,2015).

A diabetes apresenta fatores pré-existentes e condicionantes, como a obesidade, hábitos alimentares, sedentarismo, hereditariedade, entre outros fatores, e estes, em muitos casos, podem ser prevenidos ou controlados por meio de ações de promoção à saúde e mudanças nos hábitos de vida. Para Silva, torna-se imprescindível que o indivíduo participe ativamente do seu processo de tratamento de forma autônoma, para uma melhor qualidade de vida (Silva et al, 2018).

Os sujeitos que recebem o diagnóstico da síndrome tendem a tornar-se mais dependentes dos serviços de saúde, por não serem direcionados corretamente para exercer a autonomia pessoal frente ao tratamento, dessa forma inferindo diretamente na qualidade de vida do sujeito. Logo, vale ressaltar a importância de pensar não somente na prevenção, mas também em estratégias que influenciem na autonomia dos indivíduos, e por consequência em uma melhor qualidade de vida (Silva et al, 2018).

Partido desse princípio, a Atenção Básica (AB) exerce papel importante no estímulo para o empoderamento dos sujeitos no acompanhamento e tratamento da doença, por meio de ações de saúde que visem o controle da diabetes de forma efetiva. A atuação da $\mathrm{AB}$ torna-se inerente a este processo, já que se caracteriza como porta de entrada preferencial e ordenadora do cuidado nas redes de atenção à saúde, e indispensável para as estratégias de promoção da saúde, com enfoque na integralidade do sujeito (MS, 2017).

O cuidado integral à saúde incorpora novas formas nos modos de pensar e fazer o cuidado nos serviços de saúde (Assis, Nascimento, Pereira, \& Cerqueira, 2015), envolvendo ações educativas com vista ao cuidado pessoal, além de desenvolver ações que o tornem capazes de modificar a sua realidade na busca de uma melhor qualidade de vida (MS, 2006; Almeida, Moutinho \& Leite, 2016). Essa prática baseia-se na troca de saberes populares ou científicos, afim de aumentar o conhecimento dos sujeitos, estimulando sua autonomia e o autocuidado (Freire, 2018).

Neste sentido, as práticas grupais no cotidiano da AB tornam-se uma ferramenta de promoção a saúde. Estes espaços favorecem a todos os envolvidos, a valorização de diversos saberes como possibilidade terapêutica, de forma que os sujeitos sejam capazes de intervir de forma criativa no seu processo saúde-doença a partir de experiências de outras pessoas (MS, 2013). 
A definição de grupo pode ser compreendida como um conjunto de pessoas que são motivadas por necessidades semelhantes a se reunirem para uma tarefa específica, que permite a troca de dúvidas, ansiedades e inseguranças, que auxilia a lidar e a conviver melhor com a doença e favorece o sentimento de não estar sozinho no convívio com a comorbidade, o que significa, um valor terapêutico para seus participantes (Secco, Paraboni \& Arpini, 2017).

O grupo é considerado uma tecnologia de cuidado complexo, o que proporciona o desenvolvimento de ações que possibilitem a participação e corresponsabilidade do sujeito sobre sua saúde, dessa maneira, essa abordagem torna o indivíduo que convive com a diabetes, como responsável pelo seu tratamento (Silva, 2018), além de possibilitar a co-construção da autonomia pessoal por meio da interação entre as pessoas que compõe o grupo (Silva et al, 2018).

Os serviços de saúde da AB atuam na construção de uma clínica ampliada (Vieira \& Carvalho Nogueira, 2020), cujo o foco principal não está na cura da doença, e sim na compreensão de indivíduo enquanto ser biopsicossocial, e englobar a compreensão além do adoecimento, compreender o processo de convívio e a subjetividade do sujeito, relevar os determinantes sociais, bem como a inserção dos indivíduos como parte ativa em seu processo terapêutico (Chizzotti, 2014).

Diante disso, a adoção de um Grupo Estratégico de Promoção à Saúde (GEPS), é entendida como uma possibilidade de empoderamento a partir do referencial de cada sujeito, levando em consideração seus valores, crenças, hábitos e experiências de vida, auxiliam na construção de um referencial comum (Silva et al, 2018).

Dessa forma, entende-se que os grupos são espaços de crescimento, e que favorecem a prática da promoção e da educação em saúde, além dos profissionais que atuam nestes serviços passam a colaborar para o desenvolvimento da autonomia e entendimento sobre a diabetes.

Portanto, este estudo busca analisar as experiências dos sujeitos que participaram do GEPS, em relação a sua qualidade de vida, controle da doença e exercício da autonomia pessoal.

\section{Metodologia}

Este estudo faz parte de uma pesquisa maior intitulada "Grupo Estratégico de Promoção da Saúde: uma pesquisa participativa sobre a autonomia de pessoas vivendo com diabetes", que tem por objetivo desenvolver estratégias de promoção à saúde que sejam capazes de estimular a produção da autonomia pessoal, por meio da implementação de um Grupo Estratégico de Promoção da Saúde - GEPS.

Trata-se de um estudo do tipo participativo, com abordagem qualitativa, apoiada na reflexibilidade. O estudo foi realizado sob os princípios fundamentais da ética e da bioética, e aprovado pelo Comitê de Ética em Pesquisa (CEP) do Hospital Universitário Onofre Lopes (HUOL) da Universidade Federal do Rio Grande do Norte (UFRN), pelo parecer $\mathrm{N}^{\circ}$ 1.868.237 e CAAE No 61947616.4.0000.5292.

As pesquisas qualitativas abrangem diversos níveis da realidade vivenciada pelo indivíduo, trata de traços da história, da biografia, das relações, de um universo de significados, dos motivos, das aspirações, das crenças, dos valores, das atitudes e influenciam técnicas variadas do trabalho empírico (Taquette \& Minayo, 2016).

A pesquisa participativa torna-se característica ao método que foi utilizado na realização dos encontros do GEPS. Este tipo de estudo acarreta em uma potencial estratégia de mudança social, pois resulta da colaboração daqueles indivíduos envolvidos, os quais elaboram e praticam da própria mudança (Freire, 2018), além de estimular a emancipação dos sujeitos. Esse tipo de pesquisa justifica-se, ainda, por levar em consideração a produção do conhecimento compartilhado, onde os sujeitos que convivem com a doença são os coadjuvantes do estudo (Brose, 2016).

$\mathrm{O}$ caráter da reflexividade permite a não neutralidade dos pesquisadores, possibilitando uma implicação direta em conjunto com a mudança social. Nesse sentido, esta abordagem permite ao pesquisador compreender a si como objeto de estudo (Colombo, 2016). 
Dessa forma, o pesquisador enquanto participante do grupo adotou uma postura de não ofertar conhecimento técnico para o grupo, mas sim, facilitar a discussão e provocar a troca de conhecimentos horizontais entre as pessoas.

O estudo ocorreu no território sanitário da Unidade Básica de Saúde (UBS) Maria Gomes Xixi, popularmente conhecida como UBS do bairro DNER, localizada no município de Santa Cruz, interior do estado do Rio Grande do Norte, sede da V Região de Saúde, e no qual localiza-se um Campus da Universidade Federal do Rio Grande do Norte/ Faculdade de Ciências da Saúde do Trairi UFRN/FACISA - unidade de trabalho dos pesquisadores, onde outros estudos envolvendo Grupos Estratégicos de Promoção da Saúde da Pessoa com Diabetes (GEPS) são realizados.

No território da UBS existiam 54 pessoas cadastradas, com o diagnóstico do diabetes e acompanhadas pela ESF, que foram convidados para participar de um grupo do tipo fechado, dessas, 16 pessoas que vivem com diabetes foram indicadas pelos Agentes Comunitários da Saúde (ACS) para participar de 11 encontros do GEPS. Os sujeitos com Diabetes Mellitus tipo I e II foram selecionados de forma qualificada, desde que houvesse a concordância, diagnóstico prévio da síndrome, disponibilidade e interesse em fazer parte do grupo, e que estivessem vinculados a Equipe de Saúde da Família (ESF) do bairro DNER, pela característica do próprio grupo para promover sua sustentabilidade após a experiência.

A princípio houveram oito encontros, para a construção do banco dados, que consistia nas transcrições dos áudios de cada reunião, e agrupamento em narrativas. O grupo, do tipo fechado, aconteceu entre os meses de maio e agosto do ano de 2017. Estes ocorreram em uma sala reservada na UBS, com a presença de alguns Agentes Comunitários de Saúde (ACS), a enfermeira e a dentista da UBS, além do pesquisador e dos sujeitos da pesquisa. Posteriormente outros três encontros, foram realizados para validar as narrativas construídas no grupo, além de entender a gestão do mesmo, e sua permanência após a saída dos pesquisadores.

O GEPS enquanto grupo, utiliza de metodologias grupais que incentive os sujeitos participantes a pensar e refletir, por meio das próprias experiências de vida.

Os encontros tinham como base temas que eram elencados pelos próprios participantes e sistematizados anteriormente em um estudo realizado por Silva (Silva, 2014). O primeiro foi utilizado para colher pistas dos indivíduos sobre quais temas seriam abordados nas reuniões seguintes. Posteriormente todas as outras rodas de conversas tinham roteiro previamente elaborado, de acordo com as necessidades que o grupo apresentava a cada reunião realizada.

Todos os encontros do grupo foram gravados em áudios, que foram transcritos fielmente em sua versão original. Estes foram ordenadas em categorias e analisados quanto ao conteúdo, sob a perspectiva de Minayo (Minayo, 2010). As falas deram origem a narrativas, que eram lidas aos participantes do grupo e recebiam novas contribuições. Por fim, após esta análise emergiu-se os seguintes eixos temáticas: I) Reconhecer o diabete; II) Conviver com o diabetes, III) Exercitando a autonomia e o protagonismo e IV) Avaliação do grupo.

O presente estudo compreende a análise do Eixo IV "Avaliação do grupo" e dos objetivos desta pesquisa, organizouse os resultados em três categorias temáticas, sendo elas: A influência do grupo na mudança dos hábitos de vida, a importância do grupo na autonomia pessoal dos sujeitos e a influência no processo de negociação da continuidade do grupo/avaliação.

Para garantir a qualidade do trabalho, neste tipo de abordagem, foram utilizados critérios consolidados para relatos de pesquisa qualitativa (COREQ) (Souza, Marziale, Silva \& Nascimento, 2021), buscando contemplar o máximo de critérios listados no check list.

Afim de manter a privacidade do estudo, cada participante foi identificado pelo nome de uma cidade da Grécia (devido a origem do termo Diabetes), sendo elas: Véria, Atenas, Creta, Micenas, Epidauro, Metora, Zaknthos, Delfos, Rhodes, Corfu, Castória, Heraklion, Mykonos, Patras, Volos e Fira. 


\section{Resultados e Discussão}

Entre os 16 participantes do estudo, 11 eram do gênero feminino e cinco do gênero masculino, com idades entre 43 e 90 anos de idade.

\section{A influência do grupo na mudança dos hábitos de vida}

Essa categoria destaca os relatos dos sujeitos a respeito da influência da participação no grupo, e as contribuições para mudanças nos seus hábitos de vida.

As práticas grupais constituem uma importante ferramenta na $\mathrm{AB}$, no que diz respeito ao cuidado aos usuários, contribuindo para a corresponsabilização das pessoas, participação ativa, empoderamento e autonomia pessoal (MS, 2014).

A participação dos sujeitos com o mesmo diagnóstico clínico, em um grupo, cria um espaço de aprendizado, compartilhamento de experiências pessoais, além de auxiliar no processo de convívio com a doença. Dessa forma, grupos funcionam como um agente facilitador, que permite um autodesenvolvimento a respeito de como lidar com a condição de saúde (Canguilhem, 2011), por meio da partilha de vivências:

"Eu mudei [...] porque [sempre] que eu quero comer um negócio eu lembro da palestra, aí não é para eu comer isso não... ai não como". (CRETA).

"Depois que eu entrei aqui [no grupo] melhorou bastante, porque quando vocês começaram a falar [sobre os integrantes do grupo] eu comecei a ver o que deve e o que não deve, apesar de não ser tudo, mas que ajudou bastante." (PRATAS)

Creta e Patas trazem elementos que nos permitem refletir sobre a importância de compartilhar suas próprias experiências e o quanto elas, quando são horizontais, entre as pessoas de um grupo, tornam-se importantes nas suas próprias decisões.

O processo grupal, quando tem um objetivo claramente definido, com estrutura e manejo adequado para o tipo de grupo que o compõe, permite que haja uma rica troca de experiências e transformações subjetivas dos sujeitos, isso se dá, devido a miscigenação que o compõe e a possibilidade de reconhecimento no outro, que o meio favorece (MS, 2013).

A construção de saberes é influenciada por diversos elementos do cotidiano em que a pessoa vive, um grupo tem impacto significante na transformação daquelas que ali partilham, como também daqueles que os rodeiam, e de seu contexto.

Sendo assim, o grupo se caracteriza como uma fonte de apoio, onde a premissa da troca de vivências contribui significativamente no processo de transformação do indivíduo participante (Silva, 2018), de forma que considera a subjetividade de cada um durante o processo.

As condições de vida e de trabalho, graduam as mais diferentes formas de pensar e agir do indivíduo, como eles se sentem e agem a respeito do processo saúde-doença. Logo, torna-se imprescindível entender os determinantes sociais que atingem a vida daquelas pessoas, além de considerar os hábitos individuais e coletivos, em suas interações e estruturas. Dessa forma, esses processos formam polos de desempenhos semelhantes, onde os indivíduos possam ser responsáveis pelas vivências (Ayres, 2016).

Algumas complicações ocasionadas pela síndrome estão intimamente associadas aos hábitos e estilo de vida que os indivíduos praticam, é preciso compreender o processo de saúde e convívio com a doença para que haja melhor partilha de experiência (MS, 2013). 
Enfrentar a ressignificação do ser e conviver com a síndrome leva em conta costumes que precisam ser agregados ao processo. Percebe-se que, principalmente a influência de hábitos alimentares, dificulta a adaptação e constância no autocuidado, como foi expresso por Delfos:

“É uma doença que não pode comer tudo, eu sinto muita falta, mais do doce.” (DELFOS)

No entanto, destaca-se que poder ou não poder determinados alimentos é singular e deve levar em consideração cada caso, não podendo ser generalizado para todas as pessoas com diabetes.

Outra dificuldade observada nas falas dos entrevistados, foi a restrição imposta para alguns alimentos. Ragasson em seu estudo sobre a adesão ao aconselhamento nutricional em pessoas com diabetes mellitus, destacaram que a maior dificuldade enfrentada por aqueles que convivem com diabetes é a restrição alimentar, além da forma como o aconselhamento nutricional implica diretamente na adesão ou não ao tratamento não medicamentoso (Ragasson, 2016), e esse é um dos pontos que foi observado no decorrer das reuniões:

"Realmente, é uma doença muito séria, mas tem controle. Ë muito difícil você ver uma torta doce e não ter vontade de comer." (PATRAS)

"Eu não me controlo. Não existe um dia que eu diga: hoje eu não como doce! Por mais que eu tente... Às vezes, vou para um aniversário e digo: hoje, eu não vou comer. Mas, em compensação, em casa, eu já fiz a festa! Eu não me controlo! Não sei dizer o porquê. Meu “menino” traz tudo Diet, mas eu tomei abuso de coisa Diet.” (VÉRIA)

"Na diabetes, a pessoa morre pela boca. Eu sou uma." (ZAKYNTHOS)

Os valores pessoais adquiridos durante a vida, tem interferência no autocuidado com a saúde, pode-se observar que principalmente aqueles costumes que estão associados a alimentação. Nesse sentido o grupo foi capaz de, por meio da troca de vivências, auxiliar em um "novo" modelo de autocuidado, a partir do compartilhamento de saberes.

Enquanto grupo, os sujeitos compreendem que a partilha de experiências, seja ela a respeito da alimentação ou de outro método para auxílio no convívio com a diabetes, ajudam na integração da autonomia pessoal.

"O grupo é bom para trocar ideias. Tem um tipo de alimentação que usam, que poderiam compartilhar? Tem gente que faz alguma alimentação diferente, assim a gente aprende." (PATRAS).

"Ninguém é sabido para dizer que sabe de tudo. Muitas vezes, eu posso fazer algo errado achando que é certo. Nesta reunião, a gente vai trocando e aprende." (RHODES).

Dessa forma, viver em grupo é reconhecer-se nele, este dispositivo atua como estratégia de cuidado em saúde, utilizando a ideia de cogestão (Silva et al, 2018), onde o indivíduo reafirma a sua identidade como responsável por seus atos e aprende a conviver com a atual condição, por meio da co-construção, sendo capaz de desenvolver sua autonomia e protagonismo. 
Nesta categoria pode-se observar que o processo de convivência grupal com pessoas com diagnósticos similares, permite a troca de vivências, partilha de conhecimentos e percepções, além do aprendizado mútuo para melhor convivência com a síndrome, dessa maneira permitindo o gerenciamento de aprendizado e coprodução da autonomia pessoal.

\section{A importância do grupo na autonomia pessoal dos sujeitos}

Na realidade vivenciada em serviços de saúde, principalmente na $\mathrm{AB}$, pode-se observar que as pessoas que recebem o diagnóstico de diabetes em sua vida, tendem a depender mais desses serviços, pela falta de orientação quanto ao exercício da autonomia e seu protagonismo no tratamento, dessa forma acabam tendo menor qualidade de vida e empoderamento em seu tratamento (Silva et al, 2018).

"[...] quando eu queria saber como estava as taxas eu vinha aqui pedir as meninas para fazer [teste de glicemia], era dificil, mas eu vinha às vezes, quando eu fazia um exame era que sabia [...]." (EPIDAUROS)

"Quando eu descobri que tinha diabetes, logo no início, a gente pensa que é o fim do mundo, que acabou. Você pensa logo que vai morrer e começa a depender muito. No momento que a gente pensa, participa desse grupo, conversa a respeito do assunto, vai na internet, dá uma "lidazinha", a gente percebe que é algo que muita gente tem. [...]" (PATRAS).

Como observado na fala de Epidauros, o teste de glicemia é muito importante para acompanhar a sua situação de saúde, entretanto, para outros entrevistados era algo que provocavam receio, medo e insegurança, muitas vezes pela imposição por valores de referência, que poderiam estar associados ao estilo de vida por eles adotados, o que repercutia no resultado.

Logo, desmistificar o teste, e ensinar os sujeitos a realizarem sozinhos, os empodera e os torna autônomos para o realizar, cabendo então a eles a decisão de querer fazer o teste.

Reconhecer a autonomia, torna-se sempre necessário, especialmente quando há uma condição crônica de saúde, e está atrelada a práticas e gestão de cuidados com a saúde pessoal, ela é ainda mais indispensável. A Política Nacional de Humanização (MS, 2013), considera que a autonomia e protagonismo dos sujeitos, como também a corresponsabilidade entre eles no processo de autocuidado, promove um efeito significativo durante a convivência com doença, como também um empoderamento da pessoa. Essa ideia, é resguardada por meio de que qualquer alteração na saúde é mais sustentável se houver a consideração da singularidade dos indivíduos durante o processo (MS, 2013).

Logo, estratégias de autocuidado, levam em consideração a gestão, de práticas que tornem o indivíduo capaz de se sentirem habilitados a executar ações de cuidados individuais com autonomia para exercê-los.

“Com esse grupo, eu estou começando a melhorar. Eu já consegui pensar e dizer: "hoje eu vou!" Venho aqui, deixo alguém em casa, estou começando a aprender a viver, tirar um pouquinho de tempo para mim! Já estou conseguindo controlar a taxa, já perdi quatro quilos." (VÉRIA)

"A prática de exercícios, esporte, a alimentação, ter um certo controle, para que a gente consiga viver com ela. A diabetes é uma doença que a gente vai viver com ela e precisa ser feliz... tentando controlar cada vez mais, na alimentação, na educação física, no controle dos medicamentos..." (PATRAS). 
Como observado no relato de Véria e Pratas, por meio de algumas práticas, a autonomia pode ser desenvolvida, impactando dessa forma o bem-estar pessoal.

Autonomia pode ser compreendida como autodeterminação, autogoverno, capacidade de agir de maneira racional diante das opções disponíveis e possíveis, como também a de criar suas próprias condições (Freire, 2018). Nesta pesquisa consideramos que a autonomia pessoal é exercida diante de diversas experiências de vida do indivíduo, como um conceito dinâmico.

Dessa forma, o desenvolvimento da autonomia pessoal, impacta diretamente no processo de autocuidado, visto que ambos partem da perspectiva de considerar os vários fatos que constroem a vida do indivíduo, e refletem na sua forma de exercer o cuidado em saúde.

Conforme a Política Nacional de Promoção da Saúde (MS, 2015), autonomia pessoal está relacionada à identificação de potencialidades e o desenvolvimento de capacidades, dessa forma possibilitando escolhas conscientes por parte dos indivíduos. Para Paulo Freire (Freire, 2018), a autonomia é construída de acordo com várias experiências e decisões.

O GEPS, por sua característica metodológica, reforça o diálogo e compartilhamento horizontal de experiências de vida, que permitem a construção de um conhecimento mais adequado às necessidades do grupo, auxiliando na construção do conhecimento individual e coletivo, favorecendo a cogestão do cuidado.

O facilitador do grupo também possui um papel importante, estando atento aos diálogos, favorecendo o direcionamento dos objetivos de cada encontro e favorecendo uma reflexão crítica sobre o que está sendo discutido, evitando possíveis direcionamentos que podem induzir o senso comum a uma possível compreensão equivocada sobre o que se discute.

Assim, o grupo possui uma metodologia específica que auxilia na co-construção da autonomia pessoal, essa sendo compreendido como uma possível ferramenta de libertação dos indivíduos, por meio do compartilhamento de experiências com objetivos definidos.

A proposta é que o grupo possa agir como "dispositivos de ação" assim com sugere Barros (Benevides de Barros, 1996), ao se referir em grupo como um mecanismo para a coprodução, de decisões e empoderamento humano. Isso posto, o grupo funciona como um lugar para estimular a participação do indivíduo na tomada de decisões, expressão da liberdade, por conseguinte desenvolvimento da autonomia pessoal.

"[...] A questão do grupo é que a gente fica se incentivando, um incentiva o outro. Se ela faz, porque eu não posso fazer? Tem que fazer também! Todos, um incentiva o outro, um ajuda o outro...” (PATRAS).

“[...] em grupo a gente já sanou muitas dúvidas, coisas que a gente não entendia passou a entender. O grupo nos fortalece mais, a gente tem mais vontade de [...] porque [...] individualmente a gente fica "inventando" desculpas para não fazer nada e, no grupo, a gente se fortalece mais, um ajuda outro. Eu gostaria que continuasse, cada dia mais a gente vai aprendendo e vai melhorando nosso modo de vida". (CARISTO)

Assim como relatado por Pratas, o grupo tornou-se um espaço de incentivo e de compartilhamento, onde os sujeitos a partir da participação no grupo passaram a melhorar sua gestão de cuidados com a saúde, principalmente no que diz respeito a diabetes.

No grupo, o processo de coprodução da autonomia, se sujeita também ao acesso a informações e à habilidade que indivíduos poderão ter, para pensar criticamente a respeito dela (Silva et al, 2018). O GEPS se caracteriza como um dispositivo que permite a construção da autonomia pessoal das pessoas que convivem com a diabetes, por meio de troca de vivências entre sujeitos que convivem e compreendem o processo de ressignificação da síndrome em suas vidas. 
Processo de negociação para continuidade do grupo/avaliação

A cada encontro realizado, o grupo era questionado sobre a dinâmica utilizada naquele dia, e qual o aprendizado ele proporcionou. Direcionando-se para o fim do ciclo da pesquisa, devido o seu caráter participativo e de responsabilidade social, os diálogos partiram para a necessidade de continuidade do grupo.

Durante as reuniões surgiu o interesse dos próprios participantes de darem continuidade ao grupo, sendo os responsáveis diretos pelos encontros, diante do sentido que ele teria atribuído, e dos efeitos em suas vidas.

"Querendo ou não a gente pode firmar um compromisso aqui da gente continuar vindo, vai ter alguém que venha?" (DELFOS)

"Se não vier a gente em casa também pode pesquisar uma coisa, para a gente se encontrar um com os outros a gente pode fazer, independente de vir alguém, vai continuar." (PRATAS)

Assim, os três últimos encontros foram preparativos para a saída dos pesquisadores do campo e para a corresponsabilidade do grupo. Cada um destes encontros foi coordenado por um participante do grupo, para que então, a discussão desse início ao processo de gestão do grupo pelos próprios sujeitos.

Santos em seu estudo apontam que os benefícios da inclusão de práticas grupais nos planos terapêuticos das pessoas com diabetes (dos Santos Maciel et al., 2015), implicam em melhoria em diversos processos que estão diretamente associados à autonomia pessoal como, por exemplo, a diminuição da glicemia capilar, da demanda de consultas médicas, além de ser uma importante ferramenta para o fortalecimento do autocuidado (Mendonça \& Nunes, 2015).

Dessa forma, dado tais benefícios subjetivamente experimentados pelo grupo, os seus participantes tornam-se os principais interessados em ter a continuidade neste processo.

"Se eles dois não podem estar vindo agora nós assumiríamos a responsabilidade, cada um de nós assumiríamos a responsabilidade de vir, seriamos nós os professores de nós mesmos, aí com certeza nós teríamos visitas nesse encontro, mas a gente ter o compromisso da gente vir, independente se eles vão vir ou não, mas vamos ver o que aconteceu durante esses quinze dias com cada um de nós, trocando experiência, a gente pode pesquisar e com certeza vamos receber visitas..." (VOLOS)

"A gente ter um grupo no WhatsApp para diabéticos, porque é uma coisa que uma pessoa pode não ter ou não saber mexer mas tem uma filha que pode ficar interagindo para isso, aí a partir daí você passa a olhar, passa a ser mais um compromisso porque eu acho que através disso você tá longe tem uma reunião, através deste grupo você pode saber, eu acho que pode ser uma das maneiras também, não de resolver, mas de ajudar." (MICENAS)

É perceptível que o objetivo do GEPS em estimular a participação ativa no cuidado com a própria saúde por meio de práticas grupais, fora alcançado, já que os próprios sujeitos demonstraram o interesse pela metodologia e sua continuidade.

O grupo proporciona uma metodologia de aprendizagem, que implica na necessidade de alguns participantes desenvolverem uma determinada tarefa em comum, portanto o grupo torna-se um dispositivo, com o objetivo de promover saúde. 
No entanto, convém destacar que o GEPS necessita de um facilitador, não para conduzir o processo, mas para facilitá-lo, ele é fundamentado na co-gestão e não na autogestão, o que mostra a importância de implementar tais grupos na estratégia de saúde da família e coordenado pelas próprias equipes.

Assim, os GEPS funcionam como um "agente facilitador de mudança", devido a contribuição no processo de adaptação e convívio com a condição crônica que fora diagnosticado.

\section{Conclusão}

Este estudo nos permite refletir acerca das contribuições do GEPS, em relação a melhoria da qualidade de vida, controle da doença e co-produção da autonomia pessoal dos sujeitos que convivem com a diabetes. Por meio da metodologia utilizada no grupo, e os resultados expressados pelos sujeitos.

O grupo tomou uma forma de cogestão do cuidado, sendo realizado pelos próprios sujeitos, partindo do processo de reflexões acerca do cuidado do dos sujeitos de forma individual e coletiva. A partir da troca de vivências adota-se para um referencial comum, ampliando a possibilidade de decisões esclarecidas.

O GEPS influência nas mudanças de hábitos de vida dos indivíduos, levando em consideração sua subjetividade, crenças, valores, condições de trabalho, e modo de viver e reconhece a importância da autonomia pessoal na diabetes. Dessa forma, as práticas grupais como dispositivo de promoção da saúde, possibilitam um impacto positivo na vida das pessoas, desenvolvimento da autonomia e autocuidado.

Destaca-se que uma limitação deste estudo, na aplicação das metodologias propostas pelo GEPS centram-se na dificuldade de localizar os profissionais de saúde enquanto corresponsáveis pela produção do cuidado, requerendo novas estratégias para vinculá-los na atribuição de continuidade do grupo. Dessa forma, novas abordagens para que haja a sensibilização dos profissionais de saúde na adesão e continuidade das ações propostas pelo GEPS se tornariam imprescindível, na perspectiva de integrar esses profissionais aos cuidados que são exercidos pelo GEPS.

Estudos futuros com o objetivo de comprovar a efetividade das metodologias utilizadas no grupo seria viável, através da comparação de um grupo de pessoas que foram submetidas as práticas, e outro de indivíduos que não participaram dessas atividades. Dessa forma, poderíamos verificar se as experiências vividas pelos sujeitos no GEPS possuem impacto em uma melhor autonomia, controle da doença e qualidade de vida quando comparada com ao grupo que não participaram das metodologias.

\section{Agradecimentos}

Agradeço imensamente a cada sujeito participante desse estudo, que me possibilitaram crescer como pessoa e profissional, e a todos os professores e alunos envolvidos na pesquisa.

\section{Referências}

Almeida, E. R., Moutinho, C. B., \& Leite, M. T. D. S. (2016). Prática pedagógica de enfermeiros de Saúde da Família no desenvolvimento da Educação em Saúde. Interface-Comunicação, Saúde, Educação, 20, 389-402. https://doi.org/10.1590/1807-57622015.0128.

Assis, M. M. A., Nascimento, M. A. A. D., Pereira, M. J. B., \& Cerqueira, E. M. D. (2015). Cuidado integral em saúde: dilemas e desafios da enfermagem. Revista brasileira de enfermagem, 68(2), 333-338. http://dx.doi.org/10.1590/0034-7167.2015680221i.

Ayres, J. R. D. C. M. (2016). Georges Canguilhem e a construção do campo da Saúde Coletiva brasileira. Intelligere, 2(1), 139-155.

Benevides de Barros, R. D. (1997). Dispositivos em ação: o grupo. Saúde e loucura, 6, 183-91.

Brose, M. (2010). Metodologia Participativa: uma introdução a 29 instrumentos (2a ed.). Tomo Editorial.

CANGUILHEM, G. (2011). O normal e o patológico (7a ed.) Forense Universitária. 
Research, Society and Development, v. 10, n. 4, e52810414436, 2021

(CC BY 4.0) | ISSN 2525-3409 | DOI: http://dx.doi.org/10.33448/rsd-v10i4.14436

Chizzotti A. (2014). Pesquisa qualitativa em ciências humanas e sociais (6a ed.). Cortez.

Colombo, E. (2016). Reflexividade e escrita. Educação (UFSM), 1(1), 15-26. https://doi.org/10.5902/19846444420690.

dos Santos Maciel, M., Coelho, M. O., Marques, L. A. R. V., Neto, E. M. R., Lotif, M. A. L., \& Ponte, E. D. (2015). Ações de saúde desenvolvidas pelo núcleo de apoio à saúde da família-NASF. Saúde, 41(1), 117-122. http://dx.doi.org/10.5902/2236583413283.

Freire, P. (2018). Pedagogia da autonomia: Saberes necessários à prática educativa. (56a ed.), Paz e Terra.

International Diabetes Federation. Diabetes (IDF). (2015). Atlas de La Diabetes de La FID (7a ed.), Atlas.

International Diabetes Federation (IDF). (2019). Atlas De La Diabete De La FID (9a ed.), Atlas.

Mendonça, F. D. F., \& Nunes, E. D. F. P. D. A. (2015). Avaliação de grupos de educação em saúde para pessoas com doenças crônicas. Trabalho, Educação e Saúde, 13(2), 397-409. https://doi.org/10.1590/1981-7746-sip00053.

Minayo, M. C. S. (2010). O desafio do conhecimento: pesquisa qualitativa em saúde (12a ed.). Hucitec.

Ministério da Saúde - MS (2006). Caderno de Atenção Básica Diabetes Mellitus. Distrito Federal.

Ministério da Saúde - MS (2013). Estratégias para o cuidado da pessoa com doença crônica: Diabetes Mellitus Distrito Federal.

Ministério da Saúde - MS. (2014). Núcleo de Apoio à Saúde da Família: ferramentas para a gestão e para o trabalho cotidiano Distrito Federal.

Ministério da Saúde - MS. (2010). Política Nacional de Humanização: PNH. Distrito Federal.

Portaria nº 2.446, de 11 de novembro de 2014. (2014). Redefine a Política Nacional de Promoção da Saúde (PNPS).

Portaria nº 2.436, de 21 de setembro de 2017. (2017). Política Nacional de Atenção Básica (PNAB).

Ragasson, D. (2016). Fatores relacionados à adesão no tratamento do Diabetes Mellitus 2.

Secco, A. C., Paraboni, P., \& Arpini, D. M. Os Grupos como dispositivo de cuidado na AB para o trabalho com Diabéticos e Hipertensos. MudançasPsicologia da Saúde, 25(1), 9-15.

Silva, J. A. (2014). Promoção da saúde: estratégias para a autonomia e qualidade de vida do sujeito com diabetes. Dissertação de mestrado, Universidade Federal do Rio Grande do Norte, Natal, RN, Brasil.

Silva J. A. D., Souza E. C.F., Böschemeier, A. G. E., Costa C. C. M., Bezerra H. S. \& Feitosa E. E. L. C. (2018b). Diagnosis of diabetes mellitus and living with a chronic condition: participatory study. BMC Public Health, 5;18(1):699. https://doi.org/10.1186/s12889-018-5637-9.

Silva, J. A. (2018). Grupo estratégico de promoção da saúde: uma pesquisa participativa sobre a autonomia de pessoas vivendo com diabetes. Tese de doutorado, Universidade Federal do Rio Grande do Norte, Natal, RN, Brasil.

Sociedade Brasileira De Diabetes (SBD). (2020). Diretrizes Sociedade Brasileira de Diabetes 2019-2020.

Souza, V. R. D. S., Marziale, M. H. P., Silva, G. T. R., \& Nascimento, P. L. (2021). Tradução e validação para a língua portuguesa e avaliação do guia COREQ. Acta Paulista de Enfermagem, 34. https://doi.org/10.37689/acta-ape/2021ao02631.

Taquette, S. R., \& Minayo, M. C. (2016). Análise de estudos qualitativos conduzidos por médicos publicados em periódicos científicos brasileiros entre 2004 e 2013. Physis: Revista de Saúde Coletiva, 26(2), 417-434. http://dx.doi.org/10.1590/S0103-73312016000200005.

Vieira, M. M., \& de Carvalho Nogueira, C. C. (2020). A perspectiva da clínica ampliada no contexto comunitário. ANALECTA-Centro Universitário Academia, 5(5). 